\title{
Determination of the relationship between mortality and SOFA, qSOFA, MASCC scores in febrile neutropenic patients monitored in the intensive care unit
}

\author{
Tugba Cetintepe $^{1}$ (D) $\cdot$ Lutfi Cetintepe ${ }^{2}$ (D) - Serife Solmaz ${ }^{1}$ (D) $\cdot$ Sebnem Calık ${ }^{3}$ (D) $\cdot$ Mehmet Can Ugur $^{4}$.

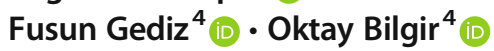

Received: 31 August 2020 / Accepted: 30 November 2020 / Published online: 6 January 2021

(C) The Author(s), under exclusive licence to Springer-Verlag GmbH, DE part of Springer Nature 2021

\begin{abstract}
Purpose Febrile neutropenia (FN) is a hematological emergency. It is challenging and confusing for the clinicians to make the decision of the febrile neutropenic patients under chemotherapy to be monitored at intensive care unit (ICU). The aim of this study was to define the factors supporting decision-making for the critical patients with febrile neutropenia.

Methods The data of 60 patients, who were taken to the ICU while they were under treatment in the Hematology Clinic with a diagnosis of febrile neutropenia, were analyzed retrospectively, in order to identify clinically useful prognostic parameters.

Results The ICU mortality rate was $80 \%$. Mortality was significantly associated with higher sequential organ failure assessment score (SOFA), quick sequential organ failure assessment score (qSOFA), and hematological SOFA (SOFAhem) scores on admission. All cases having SOFA score 10 and above and qSOFA score 2 and above died. In multivariate analysis, qSOFA score was found to be statistically significant in predicting mortality in regard to ICU admission $(p=0.004)$.

Conclusion Mortality of febrile neutropenic patients admitted to ICU is high. It would be appropriate to determine the extent of organ dysfunction instead of underlying disease, for making the decision of ICU admission. It should be noticed that the risk mortality is high for the FN cases with SOFA score 10 or above, qSOFA score 2 or above, and in need of mechanical ventilation and positive inotropic support; hence, early intervention is recommended. In our study, the most significant parameter in predicting ICU mortality was found to be qSOFA.
\end{abstract}

Keywords SOFA score $\cdot$ qSOFA score $\cdot$ Febrile neutropenia $\cdot$ Hematological malignancy $\cdot$ Intensive care unit

\section{Introduction}

Patients having hematological malignancy (HM) suffer from immunodeficiency due to the underlying disease and/or prolonged neutropenia developing after intensive chemotherapy, and hence, they are at high risk of serious infection [1]. New chemotherapeutic drugs being launched every day and becoming available for the patients have an increasing effect

Oktay Bilgir

oktaybilgir@gmail.com

1 Department of Hematology, Izmir Katip Celebi University, Ataturk Training and Research Hospital, Izmir, Turkey

2 Department of Nephrology, University of Celal Bayar, Manisa, Turkey

3 Department of Infectious Diseases \& Clinical Microbiology, Izmir Bozyaka Education and Research Hospital, University of Health Science, Izmir, Turkey

med.can@hotmail.com

Fusun Gediz

mdfusun@gmail.com
Department of Hematology, Izmir Bozyaka Education and Research Hospital, University of Health Sciences, Izmir, Turkey 
on the survival rates while severe febrile neutropenia (FN) attacks induced by resistant microorganisms limit treatment choices and increase the rate of potential complications (e.g., hypotension, acute renal failure, respiratory heart failure).

In febrile neutropenia, which is a hematological emergency, it is crucial that high-risk patients should be identified and promptly directed to the right treatment option. Although the rate of mortality in febrile neutropenia varies according to the risk group, the survival rates are quite low for the patient group monitored in the intensive care unit [2]. It is difficult to make a decision on selecting the patients that need be taken into intensive care unit and those who are eligible for further treatment in intensive care conditions. Decision-making processes should be guided by patient-based individual evaluations, case by case. Nevertheless, understanding all the other factors contributing to the prognosis will be helpful in the management of this group of patients, which is a challenging task to follow-up.

Many studies were conducted to predict mortality in febrile neutropenia and scoring systems were developed to identify the risk group. In the multinational association for supportive care in cancer (MASCC) scoring, scores are given according to the patient's age, history, symptoms, outpatient/inpatient status, and comorbid status. Patients with a MASCC score of $<21$ are considered as high-risk patients [3]. Implementation of MASCC scoring system is guiding particularly in determining where the patient will be treated. High-risk patients need hospitalization for intravenous (IV) empirical treatment. In lowrisk patients, however, oral antibiotic therapy can be used after a short inpatient period. Besides this scoring system, many national cancer associations, Infectious Diseases Society of America (IDSA) [4], National Comprehensive Cancer Network (NCCN) [5], American Society of Clinical Oncology (ASCO) [6], and European Society for Medical Oncology (ESMO) [7], have published their own guidelines on febrile neutropenia. In general, the conditions where neutropenic period is expected to continue more than 7 days are defined as high-risk conditions in these guidelines. The presence of certain types of diagnosis (acute leukemia, high-risk MDS), comorbid disease, hemodynamic instability, mucositis, gastrointestinal symptoms, new developing neurological disorder, catheter infection, pulmonary infiltration, hypoxemia, underlying lung disease, alemtuzumab use, uncontrolled progressive disease, developing liver and kidney failure, and MASCC score $<21$ was also reported as high-risk disease.

Quick sequential organ failure assessment score (qSOFA) is a scoring system that is especially useful in emergency services, developed to predict the relationship between sepsis and mortality, and it can be calculated independent of laboratory tests [8].

Sequential organ failure assessment score (SOFA) score is an effective scoring system developed to predict the survival rate in patients followed in intensive care unit, and those with organ dysfunction and failure [9]. It is very effective in determining the patients carrying high mortality risk, although it is not diagnostic for sepsis.

These scoring systems were studied in intensive care conditions for the patients having hematological malignancies [10]; however, any detailed assessment particularly for the febrile neutropenic patients, having a special place in this patient group, could not be found in the literature. The main objective of this study was to determine the mortality-related risk factors in the patient group monitored in intensive care unit with the diagnosis of FN and to investigate the effectiveness of different scoring systems for sepsis in predicting mortality in febrile neutropenia.

\section{Material and method}

The data of 60 patients, who were taken to the intensive care unit (ICU) while they were under treatment in the Hematology Clinic with a diagnosis of febrile neutropenia between January 2015 and September 2018 in the University of Health Sciences Izmir Bozyaka Education and Research Hospital, were analyzed retrospectively. Febrile neutropenia attack was defined as single temperature of $\geq 38.3^{\circ} \mathrm{C}$ or temperature sustained for at least $1 \mathrm{~h}$ at $\geq 38^{\circ} \mathrm{C}$ in patients having a neutrophil count below $500 / \mathrm{mm}^{3}$ or between 500 and $1000 / \mathrm{mm}^{3}$ with a tendency to fall below $500 / \mathrm{mm}^{3}$ within $48 \mathrm{~h}$.

Detailed patient data was obtained from electronic health records database of the hospital. Data were recorded regarding patients' demographic characteristics, type and activity of the hematological disease, length of hospital stay prior to admission to ICU, time between chemotherapy and admission to ICU, indication for ICU, need for mechanical ventilation, need for dialysis, clinical and laboratory parameters, use of vasopressors/inotropic drugs, and the microbial cultures. Disease status was determined as newly diagnosed, refractory disease, relapse, or complete partial remission according to the last bone marrow biopsy or imaging methods.

Using these data, qSOFA, SOFA, and MASCC scores were calculated for each patient at admission to the intensive care unit. SOFAhem, calculated by excluding central nervous system findings and coagulation values in patients with hematological malignancies, was also calculated for each patient at admission to the intensive care unit.

Data were analyzed using statistical package for the social sciences (SPSS) statistics 21.0 (Armonk, IBM Corp., NY, USA). Pearson's chi-square test for discrete variables or the Kruskal-Wallis test for continuous variables was used to compare patient characteristics. The Kaplan-Meier method was used to estimate internal care unit survival. We determined the optimum cutoff points for the SOFA, qSOFA, SOFAhem, and MASCC as a predictor for ICU survival based on the receiver operating characteristic (ROC) curve. The optimum cutoff points were the point on ROC curve. Gender, 
age, SOFA, qSOFA, SOFAhem, and MASCC scores all were tested in the multivariate analysis. $p<0.05$ was considered statistically significant.

\section{Ethics declarations}

The study was approved by the Institutional Review Board at University of Health Science, Izmir Bozyaka Education and Research Hospital (17/06/2020 2020-05).

\section{Results}

Sixty patients, diagnosed with febrile neutropenia and requiring intensive care, were examined in this study. The mean age was $60 \pm 15(17-85)$ years, 33 patients were male $(55 \%)$ and 27 patients were female $(45 \%)$. Most of the patients had acute leukemia (78\%), amongst which 39 patients $(65 \%)$ were diagnosed as acute myeloid leukemia (AML), 6 (10\%) as acute lymphocytic leukemia, $8(13.3 \%)$ as non-Hodgkin lymphoma, $2(3.3 \%)$ as multiple myeloma, $3(5 \%)$ as chronic lymphocytic leukemia, and $2(3.3 \%)$ as chronic myeloproliferative disease. Thirty-seven patients $(61.7 \%)$ were diagnosed with active newly diagnosed disease while 10 patients (16.7) had refractory disease, and 3 patients $(5 \%)$ developed relapse disease. Eight patients $(85 \%)$ were being monitored with complete partial remission. Fifty-one patients were determined to receive chemotherapy within the last 30 days before admitted to the ICU. Respiratory failure was the most common reason for ICU transfer, in $38.5 \%$ of the patients, followed by sepsis in $25 \%$, cardiac failure in $10 \%$, neurological reasons in $13.3 \%$, and the need for resuscitation in $13.3 \%$ of the cases. The median length of stay in the hematology clinic before admission to the ICU was 15 (2-76) days, and the median length of the hospitalization in the ICU was $3(1-20)$ days. The ICU mortality rate was $80 \%$. During patients' transfer to ICU, mean SOFA score was calculated as $12.35 \pm 4.5(2-20)$, SOFAhem score as 6.4 (1-12), qSOFA score as $2.2 \pm 1(0-$ 3 ), and MASCC score as $10.88 \pm 4.36$ (4-19). The number of patients who needed invasive mechanical ventilation in the first $24 \mathrm{~h}$ was 33 (55\%). Positive inotropic or vasopressor support was provided in $39(65 \%)$ patients. Six patients (10\%) underwent hemodialysis during follow-up in the ICU. Sterile blood, urine, and sputum cultures were taken during the febrile periods of patients, and 34 patients $(56 \%)$ had positive culture results. Twenty patients $(33.3 \%)$ had gramnegative infection, 8 patients $(13.3 \%)$ gram-positive infection, and 6 patients $(10 \%)$ had fungal infection.

The mean length of hospitalization in ICU was 4 (1-13) days for the patients over 60 years of age and 4.6 (1-20) days under 60 years. Patients over 60 years of age were observed to have a longer hospital stay $(p=0.038)$. However, age showed no effect on mortality $(p=0.42)$.
It was observed that gender, disease type and disease activity, the need for dialysis, and the length of hospital stay before admission to ICU had no effect on mortality in ICU and the length of stay in ICU.

The patients who needed mechanical ventilation at the time of admission to the ICU had a short ICU stay and this was statistically significant $(p=0.002)$. The number of patients requiring positive inotropic support was 39, and the length of hospital stay was found to be significantly short in these patients $(p=0.05)$.

As for the duration of hospitalization of the patients with positive culture results, it was observed that those with gramnegative cultures had the shortest time in the ICU, followed by those with positive fungal cultures and gram-positive cultures. It was observed that gram-negative bacterial growth significantly shortened the length of stay in ICU and all these patients died $(p=0.05)$.

The cutoff values for SOFA, SOFAhem, qSOFA, and MASCC were not uniform in the previous reports for febrile neutropenia in the internal care unit. Hence, we performed ROC curve analysis for finding optimal cutoff points for the SOFA, qSOFA, SOFAhem, and MASCC. The median SOFA was 9.5 (range 2-20), the median SOFAhem 5.5 (range 1-12), the median qSOFA was 1.5 (range $0-3$ ), and MASCC was 12.5 (range 4-19). Based on the cutoff points for the SOFA, SOFAhem, qSOFA, and MASCC, the patients were categorized into the groups as follows: high SOFA group $(\geq 9.5)$, low SOFA group (<9.5) (with a sensitivity of $83.3 \%$ and specificity of $0.00 \%$ ), high SOFAhem group $(\geq 5.5)$, low SOFA group $(<5.5)$ (with a sensitivity of $75 \%$ and specificity of $10 \%)$, high qSOFA group $(\geq 1.5)$, low qSOFA group $(<1.5)$ (with a sensitivity of $91.7 \%$ and specificity of $0.00 \%$ ), high MASCC group ( $\geq 12.5$ ), and low MASCC group $(<12.5)$ (with a sensitivity of $18.8 \%$ and specificity of $75 \%$ ).

It was observed that 12 of 20 patients with a low SOFA score survived, but all 40 patients with a high SOFA score died $(p=0.0001)$, and also, 36 of 37 patients with a high SOFAhem score died, 12 of 16 patients with a low qSOFA score survived, all of the 44 patients with a high qSOFA score died ( $p=0.0001), 3$ of 42 patients with a low MASCC score lived, and 9 of 18 patients with a high MASCC score died $(p=0.001)$.

Upon multivariate Cox regression analysis of the age, gender, SOFA, qSOFA, SOFAhem, and MASCC scores of the patients, it was determined that qSOFA score was statistically significant in predicting mortality with respect to the length of stay in ICU $(p=0.004)$.

\section{Discussion}

It is challenging and confusing for the clinicians to make the decision of the febrile neutropenic patients under 
chemotherapy to be monitored at ICU. The aim of this study was to define the factors supporting decision-making for the critical patients with febrile neutropenia.

The rates of mortality of the patients with hematologic malignity and monitored in ICU display variations with respect to treatment centers. This rate was specified as $33.7 \%$ by Bird et al. [11], as $52 \%$ by Demant et al. [12], $56 \%$ by Geers et al. [10], $60.3 \%$ by Jing Liu et al. [13], and $84.1 \%$ by Yeo et al. [14]. In some of these studies, the presence of neutropenia was observed to have an adverse effect on mortality $[13,14]$, while having no effect in some other studies. No study was encountered in the literature investigating ICU results of particularly febrile neutropenic patients. The rate of mortality of the specific patient group monitored in ICU in our study was found to be higher than the other ones, as $80 \%$. The reason of the fact that our patients below 60 years of age seem to have a higher mortality in ICU was attributed to intensive induction chemotherapy implemented to young patient group.

Monitoring acute leukemia in ICU may be rather more difficult, due to the therapy-associated complications. Contrary to what is expected, no relationship was observed in many studies between ICU mortality and underlying disease type and implemented therapy [10,15-17]. No relationship with mortality was determined in our study as well, despite high number of acute leukemia patients and their majority having had chemotherapy within 1 month prior to admission to ICU. Besides, due to the limited number of patients followed in full remission, the effect of disease status on the rate of survival in ICU could not be clearly assessed.

While Van Dijk [18] and Benoit [19] have specified a positive relationship in their studies between bacteremia and prognosis, it was observed that all our patients with gramnegative growth died during intensive care follow-up. Negative effects of gram-negative growth on the rate of mortality were supporting the study of Çalık et al. [20], in the same treatment center with our study.

The need for invasive mechanical ventilator and positive inotropic support in our study was an important determinant of mortality as it was the case in several other studies [10-15, $18]$.

Under the light of all data, in this severely critical patient group, it is rather difficult to anticipate the course of the disease by a single variable. It was confirmed in many studies that SOFA was a good indicator in $\mathrm{HM}[10,12,13]$. We have come across no study in the literature, however, conducted with febrile neutropenic patient group having hematologic malignancies, as it is the case with our patient group. Our study proved that SOFA score was a valuable indicator in anticipating prognosis in febrile neutropenic patients. Mean SOFA score of our patients calculated during their transfer to ICU was 12.35. Mean SOFA scores in HM determined by Liu ve Geerse were lower and found to be nearly $10[10$, 13]. In the study by Cornet et al., conducted with a view to anticipate ICU mortality rate in HM patients [21], it was seen that all cases with HM having SOFA score 15 and above died, and in our study, all 40 cases having SOFA score 10 and above died.

In many patients with HM, disease-associated or chemotherapy-induced thrombocytopenia can be observed. Considering that traditionally calculated SOFA score was not a sufficient indicator for these patients, Dermant et al. defined SOFAhem scoring as a more powerful marker in indicating the mortality in HM [12]. Mean SOFAhem score calculated in our FN patients was $6.4(1-12)$, which was lower than the score $(7.2 \pm 2.6)$ calculated in the study of Dermant et al. [12]. SOFAhem should be considered as a considerably quite effective scoring system in anticipating mortality in HM, and should be used in daily practice (Table 1).

The studies where bedside calculation of qSOFA score was assessed in febrile neutropenic patients were observed to be performed only in emergency departments [22, 23]. In a study where 38 patients were transferred from emergency department to ICU, qSOFA score was observed to be inadequate in predicting sepsis, mortality, and ICU requirement [22], while in another study where 25 patients were transferred from emergency department to ICU, qSOFA was negatively affecting in-hospital mortality [23]. Considerably low number of patients suggests the need for prospective studies involving larger patient populations. In our study, 60 patients monitored in hematology department and transferred to ICU were analyzed and it was found that the ones with qSOFA score 2 and above all died. This type of scoring system which can be evaluated quite fast at the bedside and without a blood test is considerably easy, and should be kept in mind by the clinicians as a repeatable method.

MASCC scoring system is a guiding method used for specifying where the outpatient febrile neutropenic cases would be receiving their first empirical antibiotic therapy. Those with MASCC $<21$ are considered as high-risk patients and recommended to get hospitalized for i.v. empirical antibiotic therapy. The data of our already hospitalized patients were evaluated with a view to compare the adequacy MASCC score in ICU follow-up decision with the other scoring systems. Mean MASCC score determined during ICU admission was 10.88 \pm 4.36 . (4-19), and MASCC score of 39 patients out of 42 patients were found as below 12. In a study conducted on 2142 patients, the relationship between MASCC score and mortality rates were evaluated, and in that study, $29 \%$ mortality was observed in the patient group with MASCC score below 15 [24]. In that study, where solid tumors were present and the majority were followed as outpatient due to low risk, mortality rates were determined to be much lower than the rates in our study, as expected.

In multivariate analysis, qSOFA score was found to be statistically significant in predicting mortality in regard to ICU admission $(p=0.004)$. 
Table 1 Comparisons of between ICU survivors and non-survivors with FN ( $n$ with $\%$ or mean $\pm \mathrm{SEM}$ )

\begin{tabular}{|c|c|c|c|c|}
\hline & Total $(n=60)$ & Survivors $(n=12)$ & Non-survivors $(n=48)$ & $p$ \\
\hline Age median [range] & $63[17-85]$ & $67[56-85]$ & $60.50[17-84]$ & $0.036^{*}$ \\
\hline \multicolumn{5}{|l|}{ Sex } \\
\hline Male & $33(55 \%)$ & $8(66.7 \%)$ & $25(52.1 \%)$ & \multirow[t]{2}{*}{0.519} \\
\hline Female & $27(45 \%)$ & $4(33.3 \%)$ & $23(47.9 \%)$ & \\
\hline \multicolumn{5}{|l|}{ Underlying disease } \\
\hline AML & $39(65 \%)$ & $8(66.7 \%)$ & $31(64.6 \%)$ & \multirow[t]{6}{*}{0.648} \\
\hline ALL & $6(10 \%)$ & $1(8.3 \%)$ & $5(10.4 \%)$ & \\
\hline NHL & $8(13.3 \%)$ & $3(2.0 \%)$ & $5(10.4 \%)$ & \\
\hline MM & $2(3.3 \%)$ & $0(0.0 \%)$ & $2(4.2 \%)$ & \\
\hline CLL & $3(5 \%)$ & $0(0.0 \%)$ & $3(6.3 \%)$ & \\
\hline CMPD & $2(3.3 \%)$ & $0(0.0 \%)$ & $2(4.2 \%)$ & \\
\hline \multicolumn{5}{|l|}{ Disease status at admission } \\
\hline Newly diagnosed & $37(61.7 \%)$ & $8(66.7 \%)$ & $29(60.4 \%)$ & \multirow[t]{5}{*}{0,942} \\
\hline Refractory disease & $10(16.7 \%)$ & $2(16.7 \%)$ & $8(16.7 \%)$ & \\
\hline Relapse & $3(5 \%)$ & $0(0.0 \%)$ & $3(6.3 \%)$ & \\
\hline Complete/partial remission & $8(13.3 \%)$ & $2(16.7 \%)$ & $6(12.5 \%)$ & \\
\hline Others & $2(3.3 \%)$ & $0(0.0 \%)$ & $2(4.2 \%)$ & \\
\hline \multicolumn{5}{|l|}{ Reason for ICU admission } \\
\hline Acute respiratory failure & $23(38.3 \%)$ & $5(41.7 \%)$ & $18(37.5 \%)$ & \multirow[t]{5}{*}{0.232} \\
\hline Heart failure & $6(10 \%)$ & $3(25.0 \%)$ & $3(5.3 \%)$ & \\
\hline Sepsis & $15(25 \%)$ & $3(25.0 \%)$ & $12(25.0 \%)$ & \\
\hline Resuscitation & $8(13.3 \%)$ & $0(0.0 \%)$ & $8(16.7 \%)$ & \\
\hline Neurological & $8(13.3 \%)$ & $1(8.3 \%)$ & $7(14.6 \%)$ & \\
\hline \multicolumn{5}{|l|}{ Chemotherapy before ICU admission } \\
\hline$\leq 30$ days & $51(85 \%)$ & $11(91.7 \%)$ & $40(83.3 \%)$ & \multirow{2}{*}{0.671} \\
\hline$>30$ days & $9(15 \%)$ & $1(8.3 \%)$ & $8(16.7 \%)$ & \\
\hline \multicolumn{5}{|l|}{ Positive culture } \\
\hline Gram-negative infection & $20(33.3 \%)$ & $0(0.0 \%)$ & $20(41.7 \%)$ & \multirow[t]{4}{*}{$0.028^{*}$} \\
\hline Gram-positive infection & $8(\% 13,3)$ & $2(\% 16,7)$ & $6(\% 12.5 \%)$ & \\
\hline Fungi infection & $6(10 \%)$ & $3(25 \%)$ & $3(6.3 \%)$ & \\
\hline None & $26(43.3 \%)$ & $7(58.3 \%)$ & $19(39.6 \%)$ & \\
\hline \multicolumn{5}{|l|}{ Cardiovascular hypotension } \\
\hline Dopamine $\leq 5$ or dobutamine(any) & $3(5 \%)$ & $0(0.0 \%)$ & $3(6.3 \%)$ & \multirow[t]{5}{*}{$0.002 *$} \\
\hline Dopamine $>5$ or $\mathrm{NE} \leq 0.1$ & $8(13.3 \%)$ & $2(16.7 \%)$ & $6(\% 12,5)$ & \\
\hline Dopamine $>15$ or $\mathrm{NE}>0.1$ & $28(46.7 \%)$ & $0(0.0 \%)$ & $28(58.3 \%)$ & \\
\hline MAP $<70$ & $6(10 \%)$ & $3(25.0 \%)$ & $3(6.3 \%)$ & \\
\hline Normal & $15(25 \%)$ & $7(58.3 \%)$ & $8(16.7 \%)$ & \\
\hline \multicolumn{5}{|l|}{ Dialysis } \\
\hline Yes & $6(10 \%)$ & $0(0 \%)$ & $6(12.5 \%)$ & \multirow[t]{2}{*}{0.333} \\
\hline No & $54(90 \%)$ & $12(100 \%)$ & $42(87.5 \%)$ & \\
\hline \multicolumn{5}{|l|}{ Invasive mechanical ventilation } \\
\hline Yes & $33(55 \%)$ & $0(0 \%)$ & $33(68.8 \%)$ & \multirow[t]{2}{*}{$0.000 *$} \\
\hline No & $27(45 \%)$ & $12(100 \%)$ & $15(31.3 \%)$ & \\
\hline SOFA score $\geq 10$ & $40(66.7 \%)$ & $0(0.0 \%)$ & $37(83.3 \%)$ & $0.001 *$ \\
\hline qSOFA score $\geq 3$ & $32(53.3 \%)$ & $0(0.0 \%)$ & $32(6.7 \%)$ & $0.001 *$ \\
\hline qSOFA score $\geq 2$ & $44(73.3 \%)$ & $0(0.0 \%)$ & $44(91.7 \%)$ & $0.001 *$ \\
\hline MASCC score $\leq 12$ & $42(70 \%)$ & $3(25.0 \%)$ & $39(81.2 \%)$ & $0.0001 *$ \\
\hline Time between hospital admission and ICU admission in days, median (range) & $15[2-76]$ & $15[2-32]$ & $16[3-76]$ & 0.296 \\
\hline Time spent in ICU in days, median (range) & $3[1-20]$ & $4[1-20]$ & $3[1-14]$ & 0.164 \\
\hline
\end{tabular}

$* p<0.05$

$A M L$, acute myelocytic leukemia; $A L L$, acute lymphoblastic leukemia; $N H L$, non-Hodgkin's lymphoma; $M M$, multiple myeloma; $C L L$, chronic lymphocytic leukemia; $C M P D$, chronic myeloproliferative disorders; $I C U$, intensive care unit; $N E$, norepinephrine; $M A P$, mean arterial pressure; SOFA, sequential organ failure assessment; $q S O F A$, quick sequential organ failure assessment; $M A S C C$, multinational association of supportive care in cancer; $F N$, febrile neutropenia

The main limitation of this study was that the number of this particular patient group was small. This was a retrospective study and we did not have patients with a comparable profile who did not die. Another limitation is this study was conducted at a single healthcare center. In other centers, decision on ICU admission may be also influenced by organizational aspects and the availability of ICU beds.
In conclusion, mortality of febrile neutropenic patients admitted to ICU is high. It would be appropriate to determine the extent of organ dysfunction instead of underlying disease, for making the decision of ICU admission. It should be noticed that the risk mortality is high for the FN cases with SOFA score 10 or above, qSOFA score 2 or above, and in need of mechanical ventilation and positive inotropic support; hence, 
early intervention is recommended. As a last remark, amongst the scores reviewed in our study, the most significant parameter in predicting ICU mortality was found to be qSOFA.

Authors' contributions Sebnem Calık had the idea for the article. The first draft of the manuscript was written by Tugba Cetintepe, and all authors commented on previous version of the manuscript. All authors read and approved the final manuscript. Statistical analysis was performed by Serife Solmaz.

Data availability I have full control of all primary data and I allow the journal to review the data if requested.

\section{Compliance with ethical standards}

Conflict of interest The authors declare that they have no conflict of interest.

Ethics approval The study was performed according to the principles of the Declaration of Helsinki, and İzmir Bozyaka Education and Research Hospital Ethics Board approved the study (approval number: 17/06/2020 2020-05).

\section{Consent to participate N/A}

\section{References}

1. Azoulay E, Pène F, Darmon $M$, Lengliné E, Benoit $D$, Soares $M$ et al (2015) Managing critically ill hematology patients: time to think differently. Blood Rev 29(6):359-367

2. Uys A, Rapoport BL, Anderson R (2004) Febrile neutropenia: a prospective study to validate the Multinational Association of Supportive Care of Cancer (MASCC) risk-index score. Support Care Cancer 12:555-560

3. Freifeld AG, Bow EJ, Sepkowitz KA et al (2011) Clinical practice guideline for the use of antimicrobial agents in neutropenic patients with cancer: 2010 update by the Infectious Diseases Society of America. Clin Infect Dis 52:e56-e93

4. Baden LR et al (2016) Prevention and treatment of cancer-related infections, version 2.2016, NCCN clinical practice guidelines in oncology. J Natl Compr Cancer Netw 14(7):882-913

5. Flowers CR, Seidenfeld J, Bow EJ et al (2013) Antimicrobial prophylaxis and outpatient management of fever and neutropenia in adults treated for malignancy: American Society of Clinical Oncology clinical practice guideline. J Clin Oncol 31(6):794-810

6. de Naurois J, Novitzky-Basso I, Gill MJ et al (2010) Management of febrile neutropenia: ESMO clinical practice guidelines. Ann Oncol 21(Suppl 5):v252-v256

7. Magid T, Haase N, Andersen JS, Nielsen OJ, Bonde J (2012) Intensive care of haematological patients. Dan Med J 59(3):A4395

8. Freund Y, Lemachatti N, Krastinova E, Van Laer M, Claessens YE, Avondo A, Occelli C, Feral-Pierssens AL, Truchot J, Ortega M, Carneiro B, Pernet J, Claret PG, Dami F, Bloom B, Riou B (2017) Beaune S; French Society of Emergency Medicine Collaborators Group. Prognostic accuracy of sepsis-3 criteria for in-hospital mortality among patients with suspected infection presenting to the emergency department. JAMA. 317(3):301-308

9. Vincent JL, de Mendonça A, Cantraine F, Moreno R, Takala J, Suter PM, Sprung CL, Colardyn F, Blecher S (1998) Use of the SOFA score to assess the incidence of organ dysfunction/failure in intensive care units: results of a multicenter, prospective study. Working group on "sepsis-related problems" of the European Society of Intensive Care Medicine. Crit Care Med 26(11):1793-1800

10. Geerse DA, Span LF, Pinto-Sietsma SJ, vanMook WN (2011) Prognosis of patients with haematological malignancies admitted to the intensive care unit: Sequential Organ Failure Assessment (SOFA) trend is a powerful predictor of mortality. Eur J Intern Med 22(1):57-61

11. Bird GT, Farquhar-Smith P, Wigmore T, Potter M, Gruber PC (2012) Outcomes and prognostic factors in patients with haematological malignancy admitted to a specialist cancer intensive care unit: a 5 yr study. Br J Anaesth 108(3):452-459

12. Demandt AMP, Geerse DA, Janssen BJP, Winkens B, Schouten HC, vanMook WNKA (2017) The prognostic value of a trend in modified SOFA score for patients with hematological malignancies in the intensive care unit. Eur J Haematol 99(4):315-322

13. Liu J, Cheng Q, Yang Q, Li X, Shen X, Zhang L, Liu Z, Khoshnood $K$ (2015) Prognosis related factors in intensive care unit (ICU) patients with hematological malignancies: a retrospective cohort analysis in a Chinese population. Hematology 20(9):494-503

14. Yeo CD, Kim JW, Kim SC, Kim YK, Kim KH, Kim HJ, Lee S, Rhee CK (2012) Prognostic factors in critically ill patients with hematologic malignancies admitted to the intensive care unit. J Crit Care 27(6):739.e1-739.e6

15. Evison J, Rickenbacher P, Ritz R, Gratwohl A, Haberthur C, Elsasser $\mathrm{S}$ et al (2001) Intensive care unit admission in patients with haematological disease: incidence, outcome and prognostic factors. Swiss Med Wkly 131:681-686

16. Massion PB, Dive AM, Doyen C, Bulpa P, Jamart J, Bosly A et al (2002) Prognosis of hematologic malignancies does not predict intensive care unit mortality. Crit Care Med 30:2260-2270

17. Kroschinsky F, Weise M, Illmer T, Haenel M, Bornhaeuser M, Hoeffken $\mathrm{G}$ et al (2002) Outcome and prognostic features of intensive care unit treatment in patients with hematological malignancies. Intensive Care Med 28:1294-1300

18. Vandijck DM, Depuydt PO, Offner FC, Nollet J, Peleman RA, Steel E, Noens LA, Decruyenaere JM, Benoit DD (2010) Impact of organ dysfunction on mortality in ICU patients with hematologic malignancies. Intensive Care Med 36(10):1744-1750

19. Benoit DD, Vandewoude KH, Decruyenaere JM, Hoste EA, Colardyn FA (2003) Outcome and early prognostic indicators in patients with a hematologic malignancy admitted to the intensive care unit for a lifethreatening complication. Crit Care Med 31(1):104-112

20. Calik S, Ari A, Bilgir O, Cetintepe T, Yis R, Sonmez U, Tosun S (2018) The relationship between mortality and microbiological parameters in febrile neutropenic patients with hematological malignancies. Saudi Med J 39(9):878-885

21. Cornet AD, Issa AI, van de Loosdrecht AA, Ossenkoppele GJ, Strackvan Schijndel RJ, Groeneveld AB (2005) Sequential organ failure predicts mortality of patients with a haematological malignancy needing intensive care. Eur J Haematol 74(6):511-516

22. Kim M, Ahn S, Kim WY, Sohn CH, Seo DW, Lee YS, Lim KS (2017) Predictive performance of the quick Sequential Organ Failure Assessment score as a screening tool for sepsis, mortality, and intensive care unit admission in patients with febrile neutropenia. Support Care Cancer 25(5):1557-1562

23. Lee SJ, Kim JH, Han SB, Paik JH, Durey A (2018) Prognostic factors predicting poor outcome in cancer patients with febrile neutropenia in the emergency department: usefulness of qSOFA. J Oncol 2018:2183179

24. Feld R (2008) Blood stream infections in cancer patients with febrile neutropenia. Int J Antimicrob Agents 32(Suppl 1):S30-S33

Publisher's note Springer Nature remains neutral with regard to jurisdictional claims in published maps and institutional affiliations. 